\title{
Review Article \\ Nanoparticle Probes for Structural and Functional Photoacoustic Molecular Tomography
}

\author{
Haobin Chen, ${ }^{1,2}$ Zhen Yuan, ${ }^{2}$ and Changfeng $W u^{1}$ \\ ${ }^{1}$ State Key Laboratory on Integrated Optoelectronics, College of Electronic Science and Engineering, Jilin University, \\ Changchun, Jilin 130012, China \\ ${ }^{2}$ Bioimaging Core, Faculty of Health Sciences, University of Macau, Taipa, Macau
}

Correspondence should be addressed to Zhen Yuan; zhenyuan@umac.mo and Changfeng Wu; cwu@jlu.edu.cn

Received 28 October 2014; Revised 11 December 2014; Accepted 11 December 2014

Academic Editor: Weibo Cai

Copyright (C) 2015 Haobin Chen et al. This is an open access article distributed under the Creative Commons Attribution License, which permits unrestricted use, distribution, and reproduction in any medium, provided the original work is properly cited.

\begin{abstract}
Nowadays, nanoparticle probes have received extensive attention largely due to its potential biomedical applications in structural, functional, and molecular imaging. In addition, photoacoustic tomography (PAT), a method based on the photoacoustic effect, is widely recognized as a robust modality to evaluate the structure and function of biological tissues with high optical contrast and high acoustic resolution. The combination of PAT with nanoparticle probes holds promises for detecting and imaging diseased tissues or monitoring their treatments with high sensitivity. This review will introduce the recent advances in the emerging field of nanoparticle probes and their preclinical applications in PAT, as well as relevant perspectives on future development.
\end{abstract}

\section{Introduction}

PAT, as an emerging powerful modality, has the capability to image the structural and functional information of biological tissues with high resolution and satisfactory imaging depth [1-4]. In PAT, a nonionizing short-pulsed laser source is generally used to illuminate the biological tissues. After the irradiated tissues absorb the light energy, the acoustic waves are produced due to thermoelastic expansion. The acoustic pressure distributions along the tissue surfaces are detected by ultrasound transducers, which can be utilized to generate the functional or structural photoacoustic images. PAT is able to image the optical properties, physiological parameters including deoxyhemoglobin and oxyhemoglobin concentrations, and mechanical parameters such as acoustic velocity of biological tissues. Interestingly photoacoustic molecular imaging can be used to detect biomarkers and reveal specific tumor cells or gene expression [5-8].

PAT based on intrinsic contrast is dominated by the endogenous agents of biological tissues such as hemoglobin, melanin, and water [9]. As a result, the imaging accuracy of PAT for diseased tissues is governed by these contrasts. For example, the diseased tissues scatter or absorb lights differently from those of healthy ones and another noticeable distinction between the two lies in respective background noise. However, the intrinsic optical contrast is probably not sufficient and in many cases may not be disease-specific. As such, it is essential to use the exogenous contrast agents which have some kinds of affinity for the diseased areas via a chemical interaction within the tissues, which is able to provide sensitive and disease-specific detection or monitoring. The use of photoacoustic exogenous agents has the capability of significantly improving the imaging sensitivity, which will greatly extend PAT's applications to areas such as deep-tissue imaging, cell-specific contrast, and molecular imaging [10-13].

In recent years nanoparticle probes have tremendously impacted scientific researches in biomedical imaging, biological diagnostics, and disease treatments [14]. In comparison with other contrast agents, nanoparticles have their very unique merits: (1) the functional properties are able to be tailored via varying their compositions, structures, sizes, and shapes; (2) the huge specific surface area as well as the quantum confinement effect in some systems bestows crucial properties for the preclinical animal study; and (3) the surface of nanoparticle probes is able to be modified to have different functions for multimodality molecular imaging. 
To date, different types of nanoparticle probes, including metal (typically Au or Ag) nanoparticles, carbon nanotubes, and superparamagnetic iron oxide nanoparticles as well as semiconductor quantum dots, have been synthesized and adopted for different biomedical applications [15-24]. PAT combined with nanoparticle contrast agents can provide unique structural and functional information at unprecedented levels. For the mini review, the recent advances in the development of nanoparticle probes in PAT are first summarized. To date, inorganic nanoparticles, such as gold nanostructures, carbon nanotubes, iron oxide, semiconductor quantum dots, and upconversion nanoparticles [20], are successfully applied in PAT as contrast agents. In addition, this work also introduces the recent progress in organic nanoparticles such as semiconducting polymer dots that show improved performance in structural and functional photoacoustic molecular tomography.

\section{Inorganic Nanoparticle Probes for PAT}

2.1. Metal Nanostructures. Metal nanoparticles have been employed for photoacoustic molecular tomography because of the biocompatibility, easy modification for targeting, minimized toxicity, and localized surface plasmon resonance peak as well as enhanced optical signals in near-infrared spectral regions [25-27]. In addition, while the intrinsic fluorescence signals from metal nanoparticles are not very strong, certain types of metal clusters and nanoparticles can be employed for fluorescent imaging or PAT. So far six types of metal nanostructures have been utilized as nanoparticle probes for PAT for in vivo tests, including nanospheres $[28,29]$, nanorods [30, 31], nanocages [32], nanoshells [33], nanobeacons [34], and nanoplates [35]. The basic preparation and in vivo and in vitro investigations for gold nanostructures in PAT have been described in detail by previous work [3]. Here we only focus on its recent advances in biomedical fields. It is widely recognized that the determination of tumor margin during the surgical resection is essential for the prevention of tumor recurrences [36, 37]. Kircher et al. have used gold nanospheres for both PAT and surfaceenhanced Raman spectroscopy (SERS). Their results revealed that multimodal molecular imaging (MRI-PAT-SERS) using the multifunctional nanoprobes can help quantify the tumor margins of living mice with high accuracy [38]. In addition, gold nanorods (GNRs) have several advantages that motivate their biomedical applications for PAT [39-41]. For example, GNRs have enhanced optical absorption cross section, which is able to generate strong photoacoustic signals and produce minimized uptakes within the reticuloendothelial systems. Accordingly, molecular imaging agents using the nanorods as a passively targeted probe were proposed, in which the probes allowed for presurgical tumor imaging for locoregional staging by PAT and intraoperative imaging of tumor margins to remove the tumors completely by SERS [31].

Nanoshells, particularly gold nanoshells, have been demonstrated as valuable vehicles for ex vivo and preclinical studies. Nanoshells coated with polyethylene glycol (PEG) can improve the biocompatibility and the circulating lifetime though the animal body. Nanoshells have been used as an intravascular contrast agent for optical coherence tomography (OCT) and PAT to identify the vascular structures of the brains [1]. Nanoshells can be conjugated to certain biomolecules that allow for specific targeting to malignant tumors because the protocol utilized to conjugate biomolecules to the surface of the gold has been well established. Nanoshells can also provide the early detection of diseases and can be used as tools for the treatment of disorders or diseases, which includes the use of hollow structures as carriers of different antitumor drugs, the development of scattering nanoshells as nanoprobes for OCT, and the implementation of absorbing nanoshells in near-infrared thermal therapy of tumors [42].

2.2. Carbon Nanotubes. Single-walled carbon nanotubes have been extensively investigated and used as nanoparticle probes for photoacoustic molecular tomography [17, 43-45]. The broad absorption spectrum of single-walled carbon nanotubes (SWCNTs) can cover the optical window of biological tissue, therefore yielding a strong photoacoustic signal. de La Zerda et al. demonstrated that SWCNTs conjugated with cyclic Arg-Gly-Asp peptides can be adopted as nanoparticle probes for photoacoustic molecular imaging of cancers [17]. The photoacoustic signals from the targeted nanotubes in mice bearing tumors were found to be eight times higher than that from the nontargeted ones. In addition, SWCNTs can be further modified to improve their performance for PAT. For instance, Indocyanine Green dye enhanced SWCNTs (SWNT-ICG) were proposed to generate photoacoustic signals that can provide a remarkably high optical contrast for in vivo animal study [21]. In particular, the ultrahigh surface area of the nanotubes has highly efficient loading of aromatic molecules such as ICG on the nanotube surface, which can create a new sort of photoacoustic probes including SWNTICG-RGD (Figure 1(a)). The optical absorption spectrum for the novel SWNT-ICG nanoparticles shows that the SWNTICG particles can perform a 20 -fold higher absorbance at its peak absorbance $(780 \mathrm{~nm})$ when compared to plain SWCNTs (Figure 1(b)). They also built a nonabsorbing and nonscattering agarose phantom with inclusions of SWNT-ICG-RGD with increased concentrations from $0.5 \mathrm{nM}$ to $121.5 \mathrm{nM}$. They found that the photoacoustic signals generated by the SWNTICG-RGD particles correlated well with the nanoparticle concentrations (Figure 1(c)). Finally, they also revealed that the new nanoparticle probes could detect cancer cells 20 times fewer than those by previously reported SWCNTs [21].

\subsection{Superparamagnetic Iron Oxide Nanoparticles. Super-} paramagnetic iron oxide nanoparticles (SPIONs) are FDA approved nanoprobes for MRI, which can also be used for nanoparticles probes for photoacoustic molecular tomography due to their perfect biosafety profiles [18, 46-49]. For example, recent work revealed that the contrast agents composed of SPIONs cores coated with silica showed their potentials as nanoparticle probes for PAT when irradiated with $1064 \mathrm{~nm}$ laser sources [46]. For this work, they employed a modulated continuous wave laser to access the maximum depth characterization of silica-coated SPION and they also tested the optical stability of the nanoprobes within different 


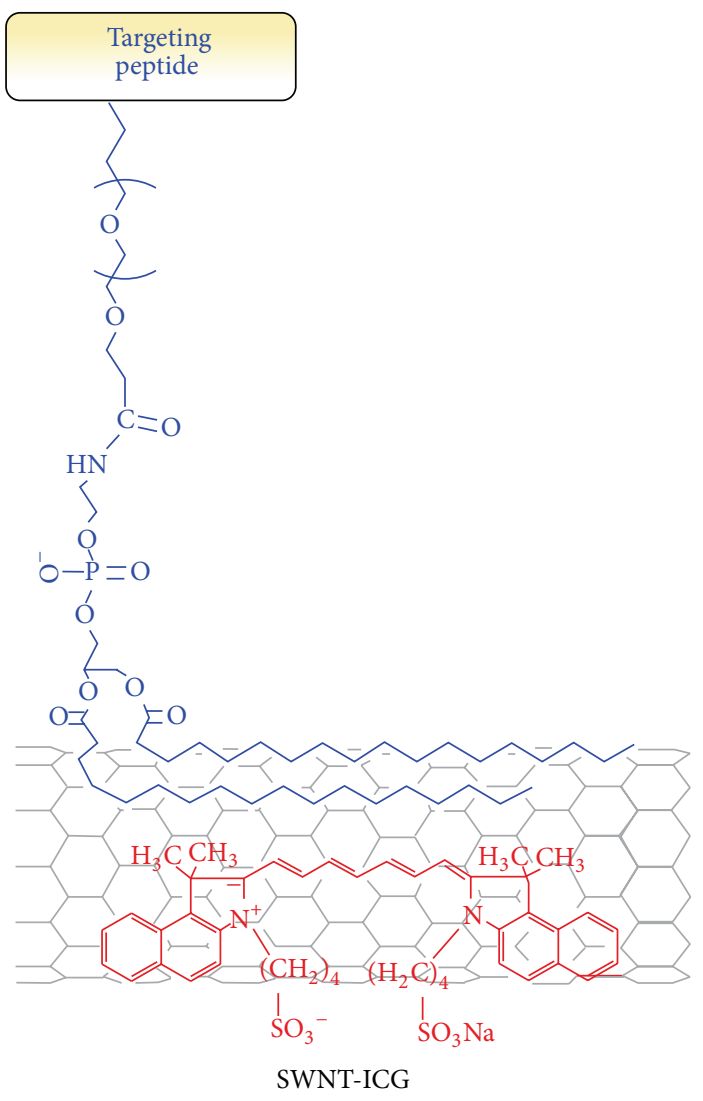

(a)

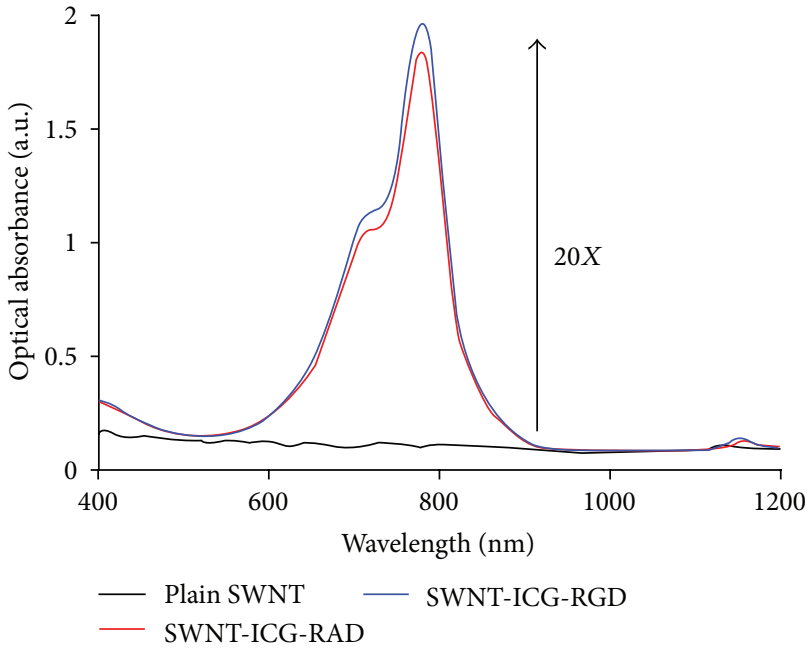

(b)

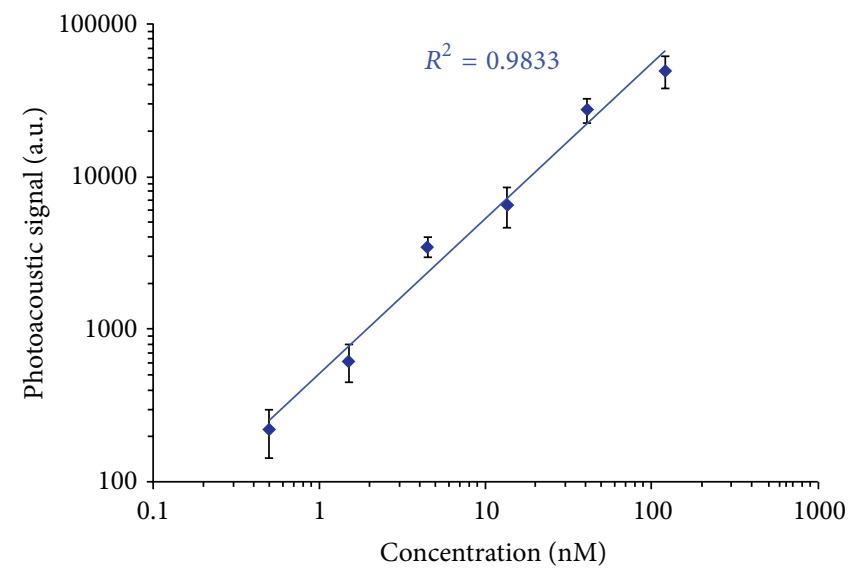

(c)

FIgURE 1: ICG dye-enhanced SWNT probes for photoacoustic imaging. (a) A schematic of a SWNT-ICG particle with ICG molecules (red) attached to the SWNT surface via noncovalent $\pi$ - $\pi$ stacking bonds. The targeting peptide was attached to SWNT via a PEGylated phospholipid. (b) The absorption spectra from different probes including SWNT-ICG-RAD (red), plain SWNT (black), and SWNT-ICGRGD (blue). The absorption intensity of ICG dye-SWNTs particles was much higher (over 20 times) than that of plain SWNT at $780 \mathrm{~nm}$. The absorption spectra between SWNT-ICG-RAD and SWNT-ICG-RGD were very similar, which validated that the peptide conjugation should not significantly perturb the photoacoustic signal. (c) The photoacoustic signals of SWNT-ICG was proportional to the probe concentrations $\left(R^{2}=0.9833\right)$. Reproduced with permission from [21].

solvents in PAT. Importantly they found that the minimum detectable concentration of the silica-coated SPION at depths of $5 \mathrm{~mm}$ and $10 \mathrm{~mm}$ inside the intralipid was $\sim 0.17$ and $\sim$ $0.23 \mathrm{mg} / \mathrm{mL}$, respectively [46].
2.4. Quantum Dots. Quantum dots (Qdots) generally refer to semiconductor nanocrystals when the physical dimensions are much smaller than the exciton Bohr radius [50, 51]. Unlike bulk counterparts, these tiny structures exhibit broad 


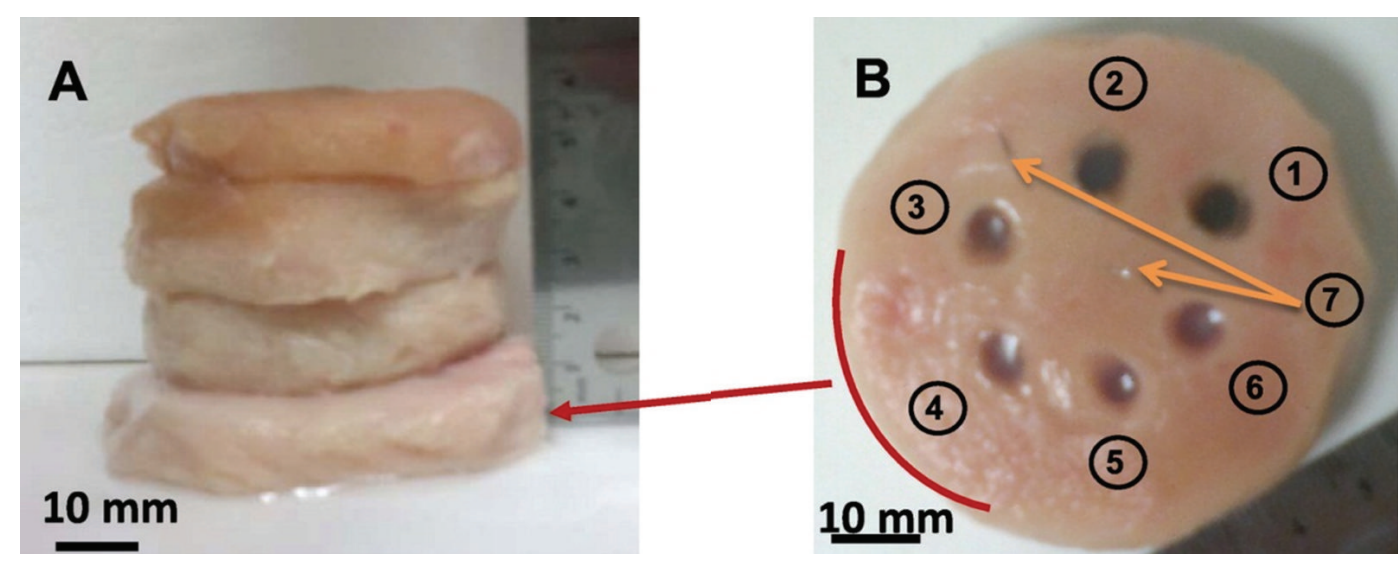

(a)

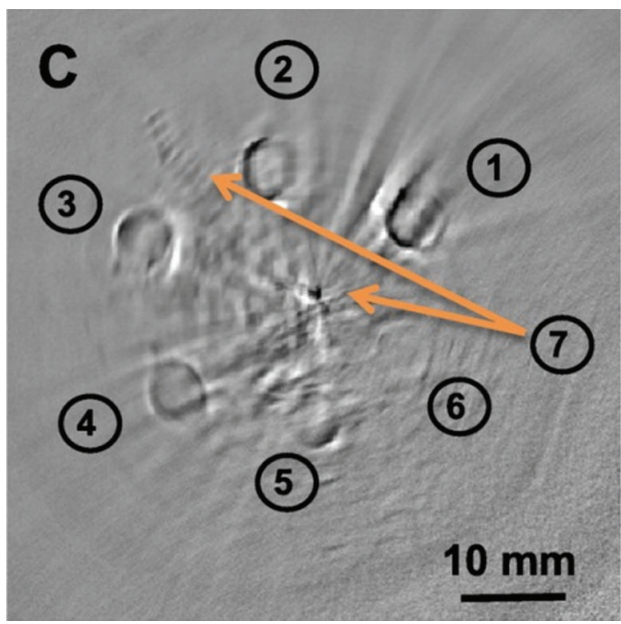

(c)

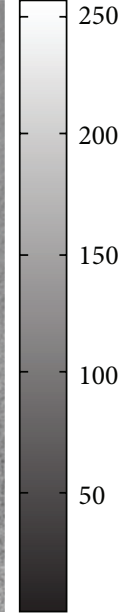

200

150

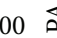

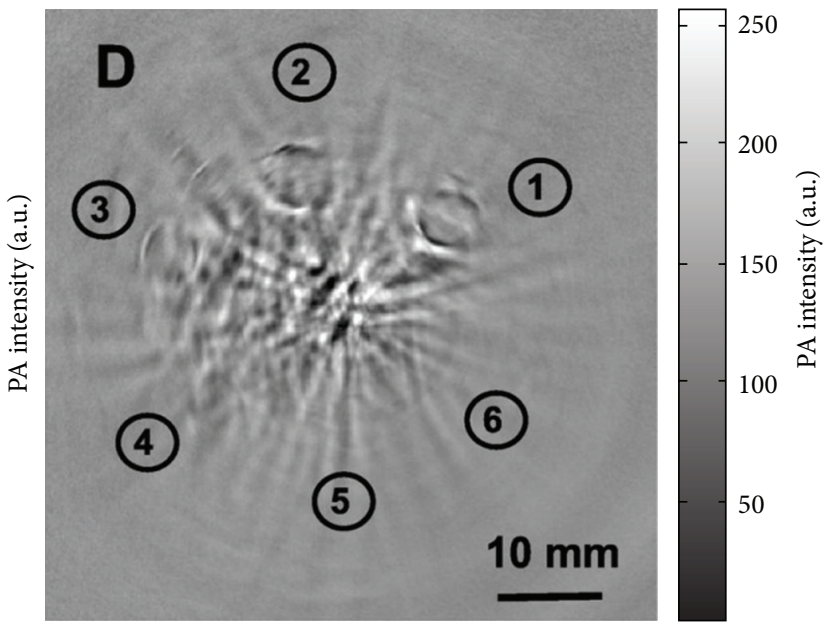

(d)

FIGURE 2: Photoacoustic molecular tomography of deep-embedded targets based on CuS Qdot probes. The agarose gels that contained CuS Qdots were placed in the background of chicken breast muscle with different chicken breasts stacked. (a) showed the configuration of chicken breast muscle stacked. (b) Photograph of chicken breast muscle that had the targets with CuS Qdots of (1) $100 \mu \mathrm{g} / \mathrm{mL}$ (2 OD), (2) $50 \mu \mathrm{g} / \mathrm{mL}$ (1 OD), (3) $25 \mu \mathrm{g} / \mathrm{mL}$ (0.5 OD), (4) $12.5 \mu \mathrm{g} / \mathrm{mL}$ (0.25 OD), (5) $6.25 \mu \mathrm{g} / \mathrm{mL}$ (0.125 OD), (6) gel without contrast agent, and (7) two needle tips at the center and 11 oclock position. The photoacoustic image was shown at the bottom low with a depth of (c) $25 \mathrm{~mm}$ and (d) $50 \mathrm{~mm}$. Reproduced with permission from [22].

excitation spectra which range from ultraviolet to nearinfrared regions and discrete energy levels that are determined primarily by their sizes and chemical compositions $[52,53]$. When compared to organic dyes or fluorescent proteins, Qdots exhibit several important properties including high absorption coefficients and enhanced photostability [54]. Due to the wide absorption bands and narrow emission bands, Qdots are considered as extraordinary contrast agents for fluorescence imaging and multiplexed detections [55]. Importantly, the large surface area of Qdots enables the design of multifunctional probes for multimodal molecular imaging. Qdots are playing an essential role as versatile labels for biomedical imaging. Interestingly, Qdots are also used as nanoparticle probes for PAT $[19,56,57]$. For example, Shashkov et al. demonstrated the applications of Qdots in PAT and photothermal microscopy [19]. By using a nanosecond pulsed laser, the bubble formation phenomena were performed using an advanced multifunctional microscope that integrated fluorescence and photoacoustic and photothermal imaging. They found that Qdots can be used as multifunctional contrast agents and sensitizers for multimodal molecular imaging and photothermal therapy [19].

Semiconductor copper sulfide $(\mathrm{CuS})$ has received extensive attention for its applications in catalysis and photovoltaics. Recently different gold nanostructures have been developed for the combination applications of PAT and chemotherapy [58, 59]. However, the near-infrared absorbance peaks of gold nanostructures, such as GNRs, would disappear after $1 \mathrm{~h}$ laser irradiation at a low power density [60]. Interestingly, the near-infrared absorption of $\mathrm{CuS}$ Qdots, derived from the d-d transition of $\mathrm{Cu}^{2+}$ ions, seldom changes with their morphologies [61]. A few modalities have been implemented for the development of CuS Qdots $[62,63]$. For example, Zhou et al. conducted 


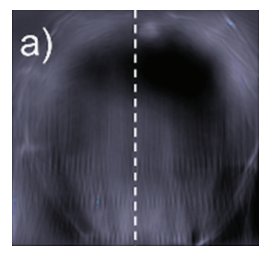

(a)

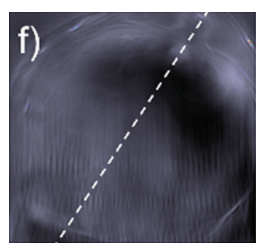

(f)

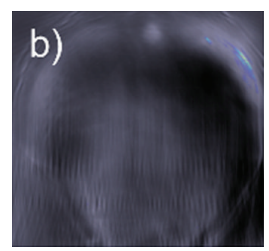

(b)

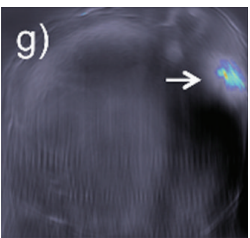

(g)

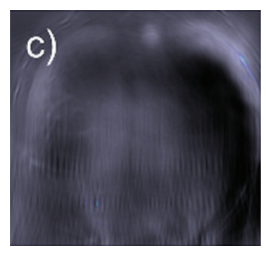

(c)

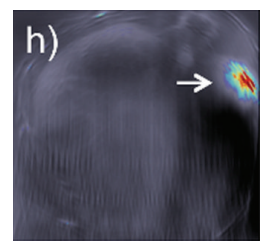

(h)

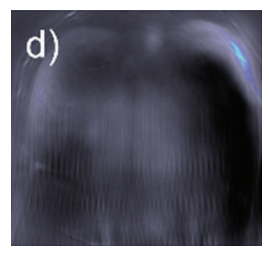

(d)

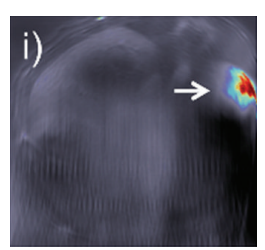

(i)

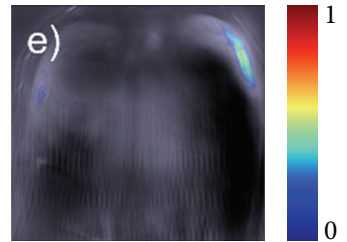

(e)

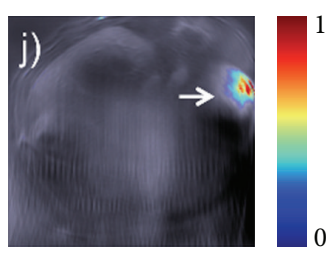

(j)

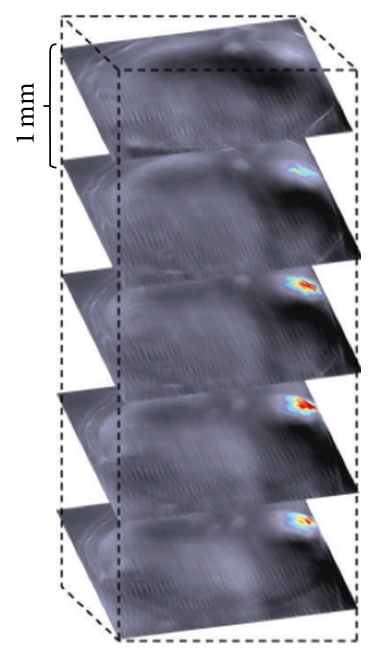

(k)

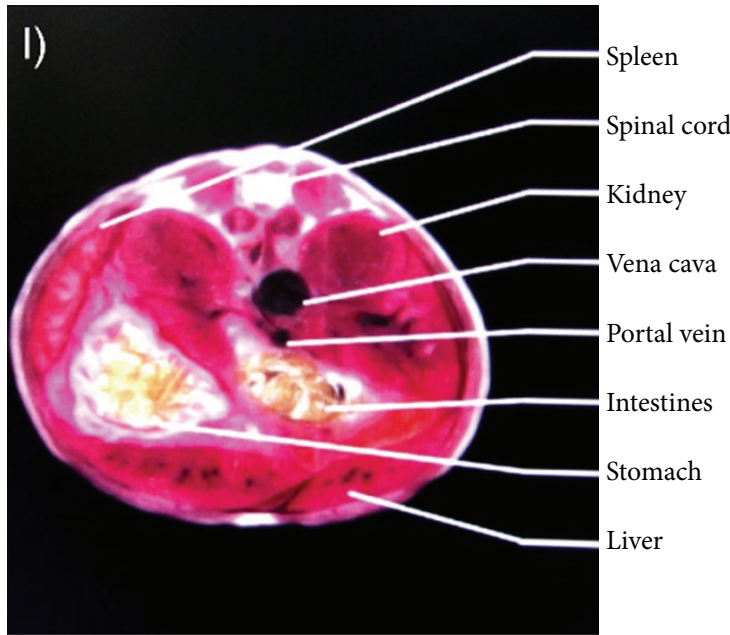

(1)

FIGURE 3: Photoacoustic molecular imaging of mice in vivo based on the lanthanide upconversion nanoparticles (UC- $\alpha$-CD). ((a)-(e)) Generated slice images of live mice before UC- $\alpha$-CD injection. ((f)-(j)) 35 minutes after injection. Dashed lines in (a) and (f) displayed positions of the mouse while (g) $-(\mathrm{j})$ showed the localized UC- $\alpha-\mathrm{CD}$. (k) 3D rendering of scanned region. (l) Schematic sections related to analysis region. Reproduced with permission from [20].

thioglycolic acid-stabilized CuS Qdots and showed their application for photothermal destruction of tumor cells in vitro using a near-infrared laser beam [64]. They also found that the absorption peak could be tuned toward longer wavelengths by simply adjusting the stoichiometric ratio between $\mathrm{CuCl}_{2}$ and $\mathrm{Na}_{2} \mathrm{~S}$ [22]. In their study, the average diameter of the CuS Qdots was $11 \mathrm{~nm}$ and the molar absorption coefficient at $1064 \mathrm{~nm}$ was estimated to be $2.6 \times 10^{7} \mathrm{~cm}^{-1} \mathrm{M}^{-1}$. The strongest absorption peak at $1064 \mathrm{~nm}$ implies that CuS Qdots are encouraging entrant for PAT contrast enhancement. Employing a Nd:YAG laser at a wavelength of $1064 \mathrm{~nm}$ for PA excitation, PAT clearly visualized CuS Qdots in mouse brain and rat lymph nodes. Furthermore, agarose gel containing CuS Qdots embedded in chicken breast at a depth of $\sim 5 \mathrm{~cm}$ could be promptly imaged with an in-plane imaging resolution of $\sim 800 \mu \mathrm{m}$ and a sensitivity of $\sim 0.7 \mathrm{nmol}$ per imaging voxel (Figure 2 ). Their work showed that it is possible to image lesions in the human breast at a depth of up to $40 \mathrm{~mm}$ with imaging resolution and sensitivity similar to that attained with CuS Qdots in chicken breast muscles. Besides the breast, lesions located in other anatomic sites such as the skin, arm or leg, head and neck, and lymph nodes may also be detected with the next generation of PAT devices equipped with more powerful $1064 \mathrm{~nm}$ lasers and a more sensitive ultrasonic detection array.

2.5. Upconversion Nanoparticles. Recently upconversion nanoparticles (UCNPs) such as $\mathrm{NaYF}_{4}$ codoped with lanthanide ions have been developed as potential nanoparticle probes for biomedical imaging $[65,66]$. Maji et al. found that the $\mathrm{NaYF}_{4}: \mathrm{Yb}^{3+}, \mathrm{Er}^{3+}$ UCNPs with $\alpha$-cyclodextrin (UC- $\alpha$-CD) in aqueous conditions were able to exhibit luminescence quenching when excited at $980 \mathrm{~nm}$. The nonradiative relaxation can result in an unprecedented and high photoacoustic signal. In vivo localization of UC- $\alpha$-CD was conducted using PAT in live mice (Figure 3) [20]. The luminescence quenching of UC- $\alpha-C D$ in aqueous solution due to nonradiative relaxation of the excited states will generate intrinsic heat 


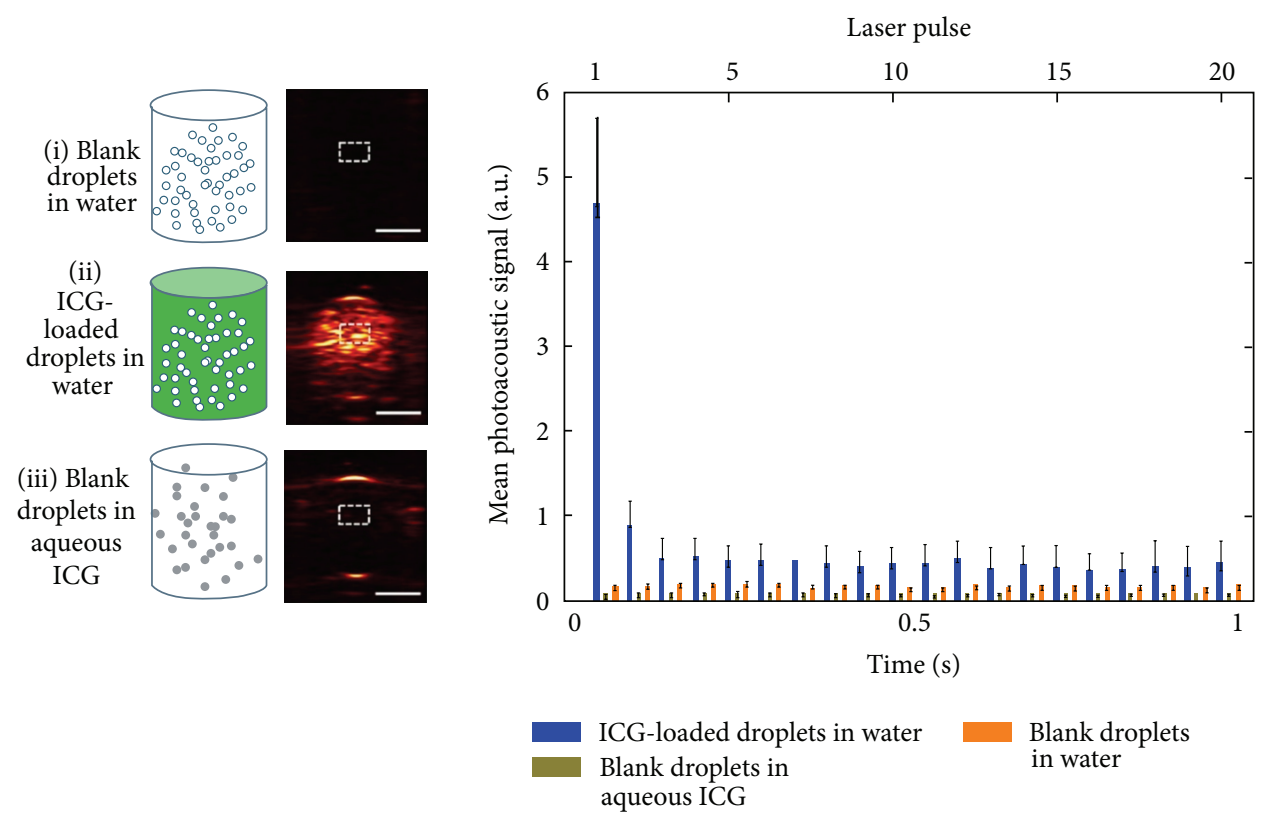

FIGURE 4: Photoacoustic molecular imaging of ICG-loaded droplets. (i) Blank droplets in water, (ii) ICG-loaded droplets in water, and (iii) blank droplets in aqueous ICG. The right panel plotted the mean photoacoustic intensity identified in the defined ROI. Error bar represents the mean \pm standard deviation. $N \geq 3$ for all reported values; scale bar $=2 \mathrm{~mm}$. Reproduced with permission from [23].

generation during the upconversion process under $980 \mathrm{~nm}$ excitation. UC- $\alpha$-CD has been identified to be noncytotoxic and suitable for in vivo PAT as shown by cytotoxicity studies. Figure 3 presented the photoacoustic images generated in real time before and $35 \mathrm{~min}$ after intravenous injection of $\mathrm{UC}-\alpha-\mathrm{CD}$ in the thoracic region of the anesthetized mouse. Contrast enhancement was observed for the images before and after injection. It was also found from the reconstructed images in Figure 3 that, compared to other contrast agents, the developed UC- $\alpha$-CD material has the advantages of easy preparation, sharp emission bandwidth, long lifetime, high photostability, low biotoxicity, and more importantly less background autofluorescence [67-70], which makes it an excellent nanoparticle probe for PAT.

\section{Organic Nanoparticle Probes for PAT}

3.1. Indocyanine Green-Loaded Photoacoustic Nanodroplets. Over the last few years, perfluorocarbon (PFC) nanodroplets have been developed into powerful probes for optical molecular imaging as well as image-guided treatments [71, 72]. For instance, high-intensity ultrasound pulses have been used to generate gas microbubbles according to the phase transitions of liquid PFC. Newly, PFC nanodroplets with encapsulated plasmonic nanoparticles were developed as probes for PAT [73]. In terms of accelerated clinical translation, Hannah et al. developed ICG-loaded PFC nanodroplets, which are identified as nontoxic, biocompatible, and safe materials [23]. The contrast enhancement via droplet vaporization was observed for PAT after the initial laser pulse, and the mean signals were determined over several pulses (Figure 4). They also evaluated the quality enhancement of PAT via the analysis of imaging contrast. Upon irradiation, the PA image contrast was $36(\mathrm{au})$, and the contrast-to-noise ratio (CNR) was $51 \mathrm{~dB}$ when compared to the $1.1(\mathrm{au})$ and $19 \mathrm{~dB}$ from blank droplets. They also investigated how the increased ambient temperature would affect the change of PAT imaging contrast. They found that, with increased temperatures, the nanodroplets will generate enhanced photoacoustic signals upon vaporization.

3.2. Semiconductor Polymer Dots. Semiconducting polymer nanoparticles (Pdots or SPNs) have recently generated tremendous interests as a novel class of contrast agents for biological imaging [74]. They exhibit several important virtues such as their extraordinary fluorescence brightness, fast emission rate, excellent photostability, and nonblinking and nontoxic features [75-81]. In particular, we recently developed several influential approaches for introducing functional groups, controlling the surface chemistry of Pdots, and employing these novel nanoparticle probes for cellular labeling and in vivo imaging [82-84]. These superior properties of Pdots over other fluorescent probes have established their enormous potential in biology and medicine as highly bright in vitro and in vivo probes.

$\mathrm{Pu}$ et al. have developed a new class of near-infrared SPN probes for in vivo photoacoustic molecular imaging [24]. SPN can generate stronger signals than SWCNTs or GNRs on a per mass basis, permitting whole-body lymph node photoacoustic mapping in living mice at a low systemic injection mass. Semiconducting polymers have been originally developed for a wide variety of optoelectronic devices. These purely organic SPNs for PAT have a unique set of advantages that derive from the light-harvesting polymers including the large mass 


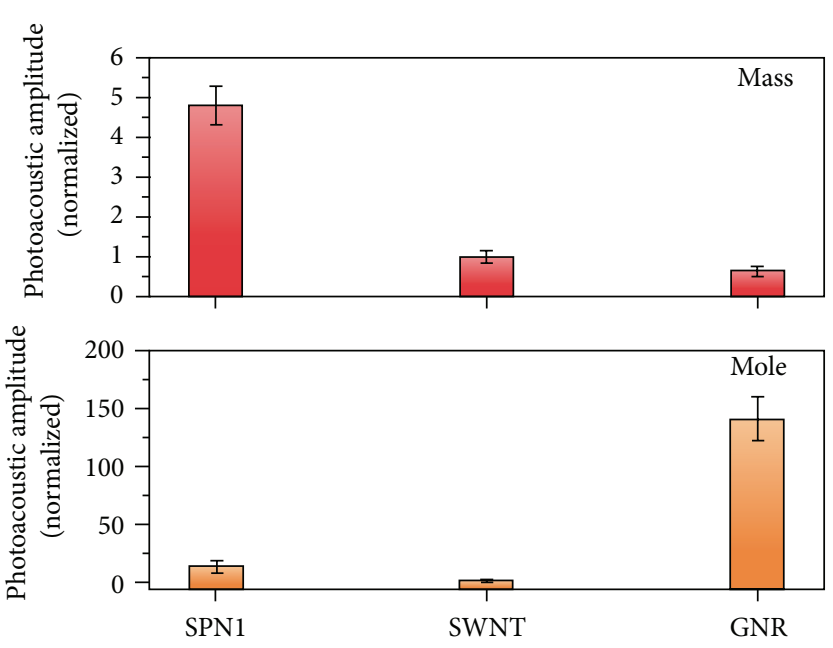

(a)

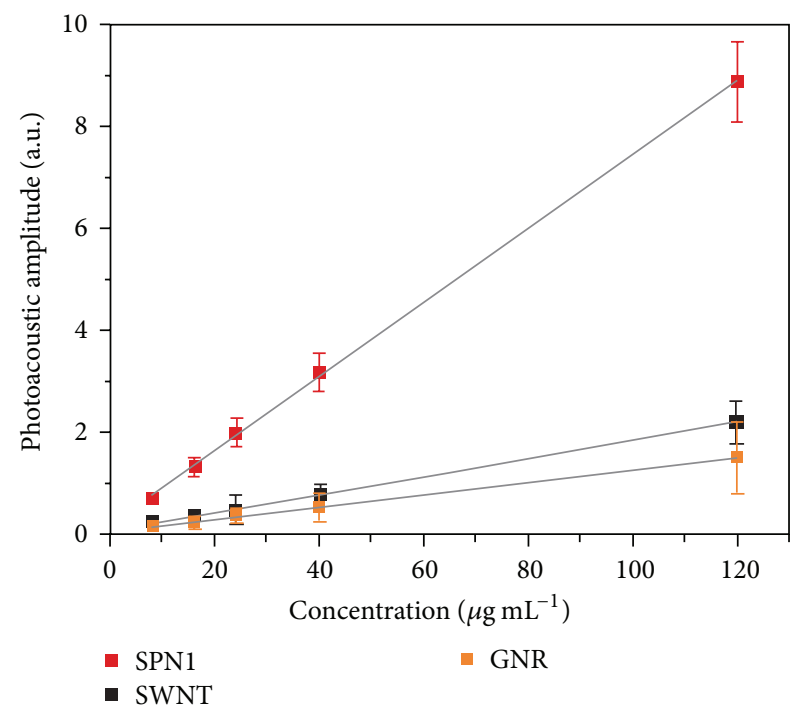

(c)

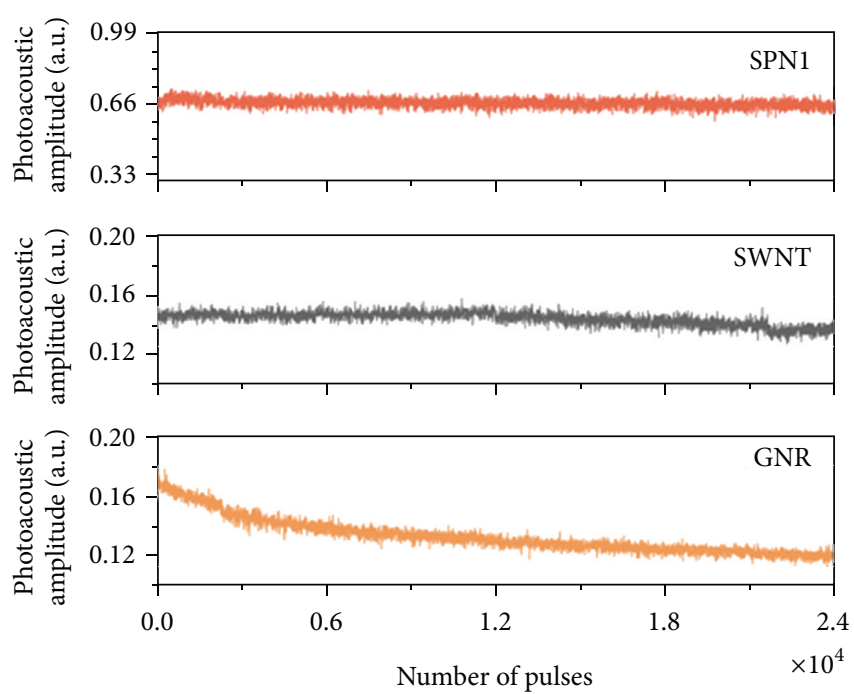

(b)
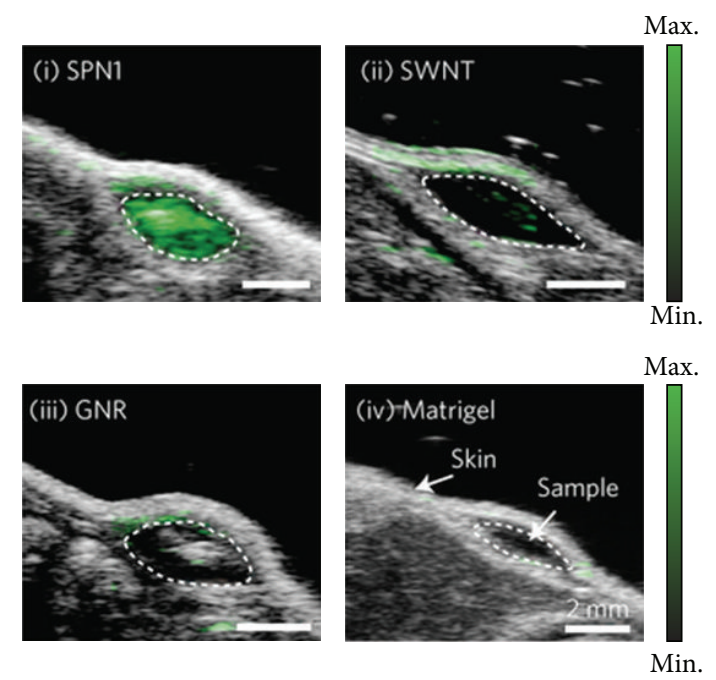

(d)

FIGURE 5: Differences on photoacoustic properties generated from conjugated polymer nanoparticles (SPN1), carbon nanotubes (SWCNTs), and gold nanorods (GNRs). (a) The photoacoustic intensity produced by different nanoparticles based on the same mass $\left(25 \mu \mathrm{g} \mathrm{mL} \mathrm{m}^{-1}\right)($ top) and molar $(48 \mathrm{nM})$ (bottom) concentrations in an agar phantom. (b) The photoacoustic intensity regarding indicated nanoparticles in agar phantoms versus the number of laser pulses. (c) The photoacoustic intensity of the nanoparticle-matrigel targets (inclusions) $(30 \mu \mathrm{L})$ in the subcutaneous dorsal space of living mice as a function of nanoparticle mass concentration. (d) The overlaid photoacoustic/ultrasound images of the nanoparticle-matrigel targets in mice at a concentration of $8 \mu \mathrm{g} \mathrm{mL}^{-1}$. Reproduced with permission from [24].

extinction coefficients and excellent photostability. These merits make SPNs superior PAT probes for generating strong and photostable photoacoustic signals in the near-infrared region when compared to SWCNT and GNR. At the same mass concentration, the intrinsic photoacoustic amplitude of the SPN at $700 \mathrm{~nm}$ can be over five times higher than that from SWCNT or GNR (Figure 5). Such high photoacoustic brightness in combination with its favorable size enables the efficient PAT of major lymph nodes in living mice with high sensitivity after a single intravenous administration of a small amount of SPNs. With the properties such as the narrow photoacoustic spectral profile, good photostability, and reactive oxygen species (ROS) inert photoacoustic signals, they further developed SPN into an activated NIR ratiometric photoacoustic probe for in vivo imaging of reaction oxygen species (ROS) (Figure 6), a hallmark of many pathological processes such as cancer, cardiomyopathy, stroke, and bacterial infections. The SPN-based photoacoustic probes effectively detect ROS and exhibit great enhancements in ratiometric photoacoustic signals $\left(\mathrm{PA}_{700} / \mathrm{PA}_{820}\right)$ of $25,7.3$, and 2.7 times in solution, in cells, and in living mice (Figure 6), respectively. 
(i) -Zymosan

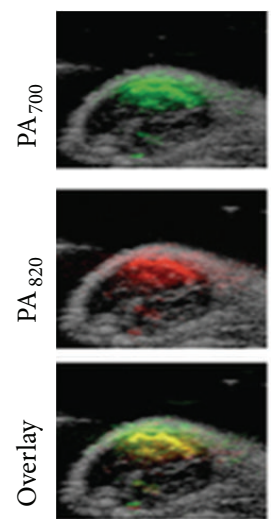

0
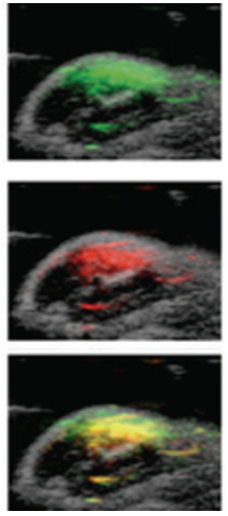

30

$(\min )$ (ii) + Zymosan
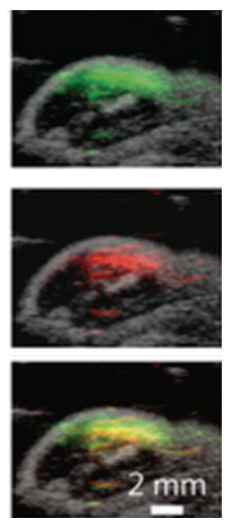

60
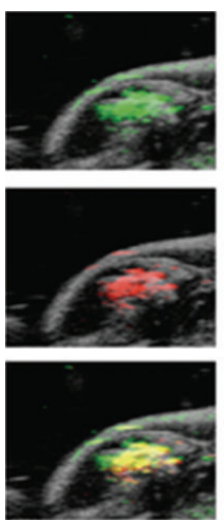

0
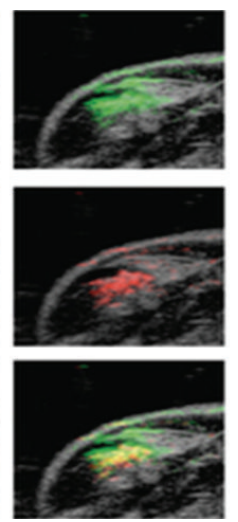

30
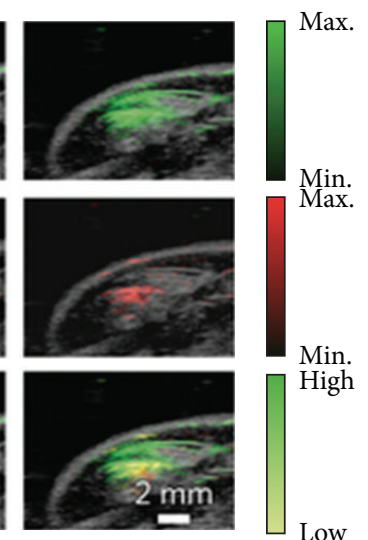

60

(a)

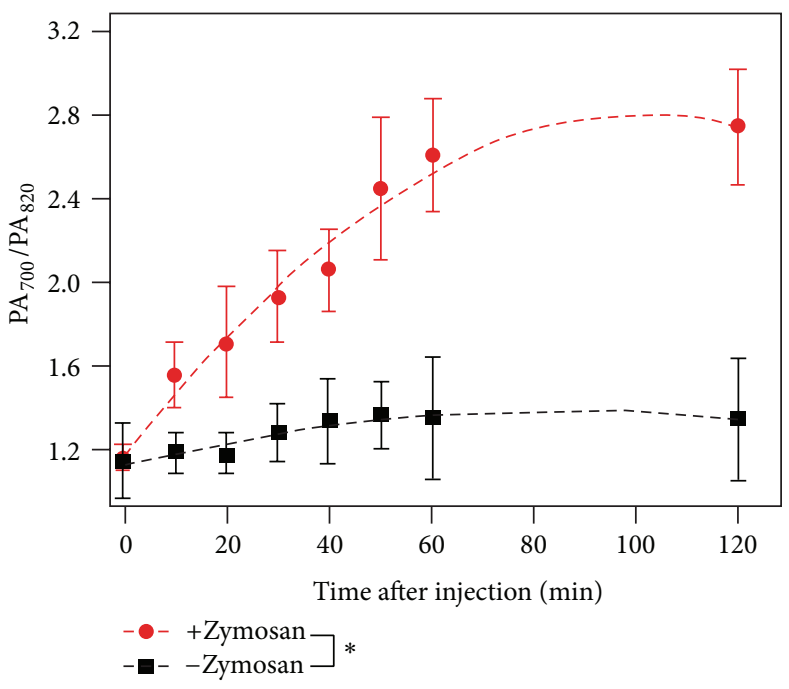

(b)

FIGURE 6: In vivo photoacoustic molecular tomography of reactive oxygen species (ROS) generation in a mouse model of acute oedema by using a ratiometric photoacoustic probe (RSPN). (a) The combined photoacoustic/ultrasound images in the thigh of living mice $(n=3)$ with respect to saline-treated (i) and zymosan-treated (ii) protocols. RSPN was injected into the thigh 20 min after zymosan treatment. (b) The ratio of photoacoustic signals generated between the wavelength of 700 and $820 \mathrm{~nm}\left(\mathrm{PA}_{700} / \mathrm{PA}_{820}\right)$ after RSPN injection. Reproduced with permission from [24].

\section{Conclusions}

In this review, recent advances of both inorganic and organic nanoparticle probes for structural and functional photoacoustic tomography have been highlighted. The use of multifarious nanoprobes for various in vitro, ex vivo, and in vivo tests represents a surging trend in nanobiotechnology and nanomedicine. However, inorganic nanoparticles have the characteristic of being nonbiodegradable, which could accumulate within the animal body for a relatively long time. Importantly, the issue on the long-term toxicity is yet not resolved for preclinical and clinical investigations. In terms of UCNPs, the efficiency of the UC process would be much lower due to the solvent relaxation problem in aqueous conditions, which should significantly affect their potential applications for in vivo photoacoustic molecular tomography. Alternatively, organic nanoparticles including semiconducting polymers show good biocompatibility as demonstrated in different cellular assays [85-87]. In the coming years, we expect a more widespread study and application of these probes with potential clinical translations, in the areas such as deep-tissue imaging, molecular diagnosis of the disease, image-guided delivery, and targeted nanoparticle drug delivery, monitoring disease progression and outcome of therapy. For the further improvement of the performance of photoacoustic probes, we envision that the development of novel light-harvesting nanoparticle species that have wellcontrolled surface properties as well as targeting capability 
and the usage of the probes for combined detection and treatment of diseases will be the most particular areas of interest in the near future.

\section{Conflict of Interests}

The authors declare that there is no conflict of interests regarding the publication of this paper.

\section{Acknowledgments}

Changfeng $\mathrm{Wu}$ acknowledges financial support from "Thousand Young Talents Program" and the National Science Foundation of China (Grants 61222508 and 61335001). The authors also acknowledge financial support from SRG201300035-FHS Grant, MYRG2014-00093-FHS and MYRG 201500036-FHS Grants from University of Macau in Macau, and FDCT Grant 026/2014/A1 from Macao Government.

\section{References}

[1] Y. Wang, X. Xie, X. Wang et al., "Photoacoustic tomography of a nanoshell contrast agent in the in vivo rat brain," Nano Letters, vol. 4, no. 9, pp. 1689-1692, 2004.

[2] L. V. Wang and S. Hu, "Photoacoustic tomography: in vivo imaging from organelles to organs," Science, vol. 335, no. 6075, pp. 1458-1462, 2012.

[3] C. Kim, C. Favazza, and L. V. Wang, "In vivo photoacoustic tomography of chemicals: high-resolution functional and molecular optical imaging at new depths," Chemical Reviews, vol. 110, no. 5, pp. 2756-2782, 2010.

[4] X. Wang, Y. Pang, G. Ku, X. Xie, G. Stoica, and L. V. Wang, "Noninvasive laser-induced photoacoustic tomography for structural and functional in vivo imaging of the brain," Nature Biotechnology, vol. 21, no. 7, pp. 803-806, 2003.

[5] Z. Yuan, C. Wu, H. Zhao, and H. Jiang, "Imaging of small nanoparticle-containing objects by finite-element-based photoacoustic tomography," Optics Letters, vol. 30, no. 22, pp. 30543056, 2005.

[6] Z. Yuan, X. Q. Li, and L. Xi, "Listening to light scattering in turbid media: quantitative optical scattering imaging using photoacoustic measurements with one-wavelength illumination," Journal of Optics, vol. 16, no. 6, Article ID 065301, 2014.

[7] Z. Yuan, J. Zhang, X. D. Wang, and C. Q. Li, "A systematic investigation of reflectance diffuse optical tomography using nonlinear reconstruction methods and continuous wave measurements," Biomedical Optics Express, vol. 5, no. 9, pp. 30113022, 2014.

[8] Z. Yuan and H. Jiang, "Simultaneous recovery of tissue physiological and acoustic properties and the criteria for wavelength selection in multispectral photoacoustic tomography," Optics Letters, vol. 34, no. 11, pp. 1714-1716, 2009.

[9] Z. Yuan and H. B. Jiang, "Photoacoustic tomography for imaging nanoparticles," in Cancer Nanotechnology, S. R. Grobmyer and B. Moudgil, Eds., vol. 624 of Methods in Molecular Biology, Humana Press, 2010.

[10] L. Nie, S. Wang, X. Wang et al., "In vivo volumetric photoacoustic molecular angiography and therapeutic monitoring with targeted plasmonic nanostars," Small, vol. 10, no. 8, pp. 15851593, 2014.
[11] J. R. Cook, W. Frey, and S. Emelianov, "Quantitative photoacoustic imaging of nanoparticles in cells and tissues," ACS Nano, vol. 7, no. 2, pp. 1272-1280, 2013.

[12] X. Cai, W. Li, C.-H. Kim, Y. Yuan, L. V. Wang, and Y. Xia, “In vivo quantitative evaluation of the transport kinetics of gold nanocages in a lymphatic system by noninvasive photoacoustic tomography," ACS Nano, vol. 5, no. 12, pp. 9658-9667, 2011.

[13] Z. Chen, L. Ma, Y. Liu, and C. Chen, "Applications of functionalized fullerenes in tumor theranostics," Theranostics, vol. 2, no. 3, pp. 238-250, 2012.

[14] H. Xu, Q. Li, L. Wang et al., "Nanoscale optical probes for cellular imaging," Chemical Society Reviews, vol. 43, no. 8, pp. 2650-2661, 2014.

[15] Q. Zhang, N. Iwakuma, P. Sharma et al., "Gold nanoparticles as a contrast agent for in vivo tumor imaging with photoacoustic tomography," Nanotechnology, vol. 20, no. 39, Article ID 395102, 2009.

[16] K. A. Homan, M. Souza, R. Truby et al., "Silver nanoplate contrast agents for in vivo molecular photoacoustic imaging," ACS Nano, vol. 6, no. 1, pp. 641-650, 2012.

[17] A. de La Zerda, C. Zavaleta, S. Keren et al., "Carbon nanotubes as photoacoustic molecular imaging agents in living mice," Nature Nanotechnology, vol. 3, no. 9, pp. 557-562, 2008.

[18] L. Xi, S. R. Grobmyer, G. Zhou, W. Qian, L. Yang, and H. Jiang, "Molecular photoacoustic tomography of breast cancer using receptor targeted magnetic iron oxide nanoparticles as contrast agents," Journal of Biophotonics, vol. 7, no. 6, pp. 401-409, 2014.

[19] E. V. Shashkov, M. Everts, E. I. Galanzha, and V. P. Zharov, "Quantum dots as multimodal photoacoustic and photothermal contrast agents," Nano Letters, vol. 8, no. 11, pp. 3953-3958, 2008.

[20] S. K. Maji, S. Sreejith, J. Joseph et al., "Upconversion nanoparticles as a contrast agent for photoacoustic imaging in live mice," Advanced Materials, vol. 26, no. 32, pp. 5633-5638, 2014.

[21] A. D. L. Zerda, Z. Liu, S. Bodapati et al., "Ultrahigh sensitivity carbon nanotube agents for photoacoustic molecular imaging in living mice," Nano Letters, vol. 10, no. 6, pp. 2168-2172, 2010.

[22] G. Ku, M. Zhou, S. Song, Q. Huang, J. Hazle, and C. Li, “Copper sulfide nanoparticles as a new class of photoacoustic contrast agent for deep tissue imaging at $1064 \mathrm{~nm}$," ACS Nano, vol. 6, no. 8, pp. 7489-7496, 2012.

[23] A. Hannah, G. Luke, K. Wilson, K. Homan, and S. Emelianov, "Indocyanine green-loaded photoacoustic nanodroplets: dual contrast nanoconstructs for enhanced photoacoustic and ultrasound imaging," ACS Nano, vol. 8, no. 1, pp. 250-259, 2014.

[24] K. Y. Pu, A. J. Shuhendler, J. V. Jokerst et al., "Semiconducting polymer nanoparticles as photoacoustic molecular imaging probes in living mice," Nature Nanotechnology, vol. 9, no. 3, pp. 233-239, 2014.

[25] C. Fan, S. Wang, J. W. Hong, G. C. Bazan, K. W. Plaxco, and A. J. Heeger, "Beyond superquenching: hyper-efficient energy transfer from conjugated polymers to gold nanoparticles," Proceedings of the National Academy of Sciences of the United States of America, vol. 100, no. 11, pp. 6297-6301, 2003.

[26] C. J. Murphy, A. M. Gole, J. W. Stone et al., "Gold nanoparticles in biology: beyond toxicity to cellular imaging," Accounts of Chemical Research, vol. 41, no. 12, pp. 1721-1730, 2008.

[27] J. Chen, F. Saeki, B. J. Wiley et al., "Gold nanocages: bioconjugation and their potential use as optical imaging contrast agents," Nano Letters, vol. 5, no. 3, pp. 473-477, 2005.

[28] W. Lu, Q. Huang, G. Ku et al., "Photoacoustic imaging of living mouse brain vasculature using hollow gold nanospheres," Biomaterials, vol. 31, no. 9, pp. 2617-2626, 2010. 
[29] W. Lu, M. P. Melancon, C. Xiong et al., "Effects of photoacoustic imaging and photothermal ablation therapy mediated by targeted hollow gold nanospheres in an orthotopic mouse xenograft model of glioma," Cancer Research, vol. 71, no. 19, pp. 6116-6121, 2011.

[30] J. V. Jokerst, M. Thangaraj, P. J. Kempen, R. Sinclair, and S. S. Gambhir, "Photoacoustic imaging of mesenchymal stem cells in living mice via silica-coated gold nanorods," ACS Nano, vol. 6, no. 7, pp. 5920-5930, 2012.

[31] J. V. Jokerst, A. J. Cole, D. van de Sompel, and S. S. Gambhir, "Gold nanorods for ovarian cancer detection with photoacoustic imaging and resection guidance via Raman imaging in living mice," ACS Nano, vol. 6, no. 11, pp. 10366-10377, 2012.

[32] E. C. Cho, C. Kim, F. Zhou et al., "Measuring the optical absorption cross sections of $\mathrm{Au}-\mathrm{Ag}$ nanocages and au nanorods by photoacoustic imaging," The Journal of Physical Chemistry C, vol. 113, no. 21, pp. 9023-9028, 2009.

[33] H. J. Lee, Y. Liu, J. Zhao et al., "In vitro and in vivo mapping of drug release after laser ablation thermal therapy with doxorubicin-loaded hollow gold nanoshells using fluorescence and photoacoustic imaging," Journal of Controlled Release, vol. 172, no. 1, pp. 152-158, 2013.

[34] D. Pan, M. Pramanik, A. Senpan et al., "Molecular photoacoustic imaging of angiogenesis with integrin-targeted gold nanobeacons," The FASEB Journal, vol. 25, no. 3, pp. 875-882, 2011.

[35] G. P. Luke, A. Bashyam, K. A. Homan, S. Makhija, Y.-S. Chen, and S. Y. Emelianov, "Silica-coated gold nanoplates as stable photoacoustic contrast agents for sentinel lymph node imaging," Nanotechnology, vol. 24, no. 45, Article ID 455101, 2013.

[36] M. K. Bucci, A. Maity, A. J. Janss et al., "Near complete surgical resection predicts a favorable outcome in pediatric patients with nonbrainstem, malignant gliomas: results from a single center in the magnetic resonance imaging era," Cancer, vol. 101, no. 4 , pp. 817-824, 2004.

[37] R. Stupp, M. E. Hegi, M. J. van den Bent et al., "Changing paradigms - an update on the multidisciplinary management of malignant glioma," Oncologist, vol. 11, no. 2, pp. 165-180, 2006.

[38] M. F. Kircher, A. de La Zerda, J. V. Jokerst et al., "A brain tumor molecular imaging strategy using a new triple-modality MRIphotoacoustic-Raman nanoparticle," Nature Medicine, vol. 18, no. 5, pp. 829-834, 2012.

[39] G. von Maltzahn, J.-H. Park, A. Agrawal et al., "Computationally guided photothermal tumor therapy using long-circulating gold nanorod antennas," Cancer Research, vol. 69, no. 9, pp. 3892-3900, 2009.

[40] K. Kim, S.-W. Huang, S. Ashkenazi et al., "Photoacoustic imaging of early inflammatory response using gold nanorods," Applied Physics Letters, vol. 90, no. 22, Article ID 223901, 2007.

[41] T. B. Huff, M. N. Hansen, Y. Zhao, J.-X. Cheng, and A. Wei, "Controlling the cellular uptake of gold nanorods," Langmuir, vol. 23, no. 4, pp. 1596-1599, 2007.

[42] C. Loo, A. Lin, L. Hirsch et al., "Nanoshell-enabled photonicsbased imaging and therapy of cancer," Technology in Cancer Research \& Treatment, vol. 3, no. 1, pp. 33-40, 2004.

[43] L. Xiang, Y. Yuan, D. Xing, Z. Ou, S. Yang, and F. Zhou, "Photoacoustic molecular imaging with antibody-functionalized single-walled carbon nanotubes for early diagnosis of tumor," Journal of Biomedical Optics, vol. 14, no. 2, Article ID 021008, 2009.
[44] J.-W. Kim, E. I. Galanzha, E. V. Shashkov, H.-M. Moon, and V. P. Zharov, "Golden carbon nanotubes as multimodal photoacoustic and photothermal high-contrast molecular agents," Nature Nanotechnology, vol. 4, no. 10, pp. 688-694, 2009.

[45] F. Zhou, S. Wu, Y. Yuan, W. R. Chen, and D. Xing, "Mitochondria-targeting photoacoustic therapy using singlewalled carbon nanotubes," Small, vol. 8, no. 10, pp. 1543-1550, 2012.

[46] R. Alwi, S. Telenkov, A. Mandelis et al., "Silica-coated super paramagnetic iron oxide nanoparticles (SPION) as biocompatible contrast agent in biomedical photoacoustics," Biomedical Optics Express, vol. 3, no. 10, pp. 2500-2509, 2012.

[47] D. J. Grootendorst, J. Jose, R. M. Fratila et al., "Evaluation of superparamagnetic iron oxide nanoparticles (Endorem) as a photoacoustic contrast agent for intra-operative nodal staging," Contrast Media and Molecular Imaging, vol. 8, no. 1, pp. 83-91, 2013.

[48] X. Feng, F. Gao, and Y. Zheng, "Thermally modulated photoacoustic imaging with super-paramagnetic iron oxide nanoparticles," Optics Letters, vol. 39, no. 12, pp. 3414-3417, 2014.

[49] R. Qiao, C. Yang, and M. Gao, "Superparamagnetic iron oxide nanoparticles: from preparations to in vivo MRI applications," Journal of Materials Chemistry, vol. 19, no. 35, pp. 6274-6293, 2009.

[50] A. P. Alivisatos, "Semiconductor clusters, nanocrystals, and quantum dots," Science, vol. 271, no. 5251, pp. 933-937, 1996.

[51] W. C. W. Chan, D. J. Maxwell, X. Gao, R. E. Bailey, M. Han, and S. Nie, "Luminescent quantum dots for multiplexed biological detection and imaging," Current Opinion in Biotechnology, vol. 13, no. 1, pp. 40-46, 2002.

[52] A. M. Smith and S. M. Nie, "Semiconductor nanocrystals: structure, properties, and band gap engineering," Accounts of Chemical Research, vol. 43, no. 2, pp. 190-200, 2010.

[53] C. B. Murray, D. J. Norris, and M. G. Bawendi, "Synthesis and characterization of nearly monodisperse $\mathrm{CdE}(\mathrm{E}=$ sulfur, selenium, tellurium) semiconductor nanocrystallites," Journal of the American Chemical Society, vol. 115, no. 19, pp. 8706-8715, 1993.

[54] X. Gao, L. Yang, J. A. Petros, F. F. Marshall, J. W. Simons, and $\mathrm{S}$. Nie, "In vivo molecular and cellular imaging with quantum dots," Current Opinion in Biotechnology, vol. 16, no. 1, pp. 63-72, 2005.

[55] L. A. Bentolila, Y. Ebenstein, and S. Weiss, "Quantum dots for in vivo small-animal imaging," The Journal of Nuclear Medicine, vol. 50, no. 4, pp. 493-496, 2009.

[56] T. Toyoda, K. Oshikane, D. Li, Y. Luo, Q. Meng, and Q. Shen, "Photoacoustic and photoelectrochemical current spectra of combined CdS/CdSe quantum dots adsorbed on nanostructured $\mathrm{TiO}_{2}$ electrodes, together with photovoltaic characteristics," Journal of Applied Physics, vol. 108, no. 11, Article ID 114304, 2010.

[57] S. Abdallah, N. Al-Hosiny, and A. Badawi, "Photoacoustic study of CdS QDs for application in quantum-dot-sensitized solar cells," Journal of Nanomaterials, vol. 2012, Article ID 498286, 6 pages, 2012.

[58] P. Huang, J. Lin, W. Li et al., "Biodegradable gold nanovesicles with an ultrastrong plasmonic coupling effect for photoacoustic imaging and photothermal therapy," Angewandte ChemieInternational Edition, vol. 52, no. 52, pp. 13958-13964, 2013. 
[59] H.-W. Yang, H.-L. Liu, M.-L. Li et al., "Magnetic goldnanorod/PNIPAAmMA nanoparticles for dual magnetic resonance and photoacoustic imaging and targeted photothermal therapy," Biomaterials, vol. 34, no. 22, pp. 5651-5660, 2013.

[60] K. Yang, H. Xu, L. Cheng, C. Sun, J. Wang, and Z. Liu, "In vitro and in vivo near-infrared photothermal therapy of cancer using polypyrrole organic nanoparticles," Advanced Materials, vol. 24, no. 41, pp. 5586-5592, 2012.

[61] Y. Li, W. Lu, Q. Huang, C. Li, and W. Chen, "Copper sulfide nanoparticles for photothermal ablation of tumor cells," Nanomedicine, vol. 5, no. 8, pp. 1161-1171, 2010.

[62] L. Xu, X. Chen, L. Ma, and F. Gao, "Facile preparation of copper sulfide nanoparticles from perovskite templates containing bromide anions," Colloids and Surfaces A: Physicochemical and Engineering Aspects, vol. 349, no. 1-3, pp. 69-73, 2009.

[63] P. Huang, Z. Li, H. Hu, and D. Cui, "Synthesis and characterization of bovine serum albumin-conjugated copper sulfide nanocomposites," Journal of Nanomaterials, vol. 2010, Article ID 641545, 6 pages, 2010.

[64] M. Zhou, R. Zhang, M. Huang et al., "A chelator-free multifunctional $\left[{ }^{64} \mathrm{Cu}\right] \mathrm{CuS}$ nanoparticle platform for simultaneous micro-PET/CT imaging and photothermal ablation therapy," Journal of the American Chemical Society, vol. 132, no. 43, pp. 15351-15358, 2010.

[65] Z. Li, Y. Zhang, and S. Jiang, "Multicolor core/shell-structured upconversion fluorescent nanoparticles," Advanced Materials, vol. 20, no. 24, pp. 4765-4769, 2008.

[66] F. Vetrone, R. Naccache, A. J. de La Fuente et al., "Intracellular imaging of HeLa cells by non-functionalized $\mathrm{NaYF}_{4}: \mathrm{Er}^{3+}, \mathrm{Yb}^{3+}$ upconverting nanoparticles," Nanoscale, vol. 2, no. 4, pp. 495498, 2010

[67] N.-N. Dong, M. Pedroni, F. Piccinelli et al., "NIR-to-NIR twophoton excited $\mathrm{CaF}_{2}: \mathrm{Tm}^{3+}, \mathrm{Yb}^{3+}$ nanoparticles: multifunctional nanoprobes for highly penetrating fluorescence bio-imaging," ACS Nano, vol. 5, no. 11, pp. 8665-8671, 2011.

[68] Q. Liu, T. Yang, W. Feng, and F. Li, "Blue-emissive upconversion nanoparticles for low-power-excited bioimaging in vivo," Journal of the American Chemical Society, vol. 134, no. 11, pp. 5390-5397, 2012.

[69] Y. Yang, Q. Shao, R. Deng et al., "In vitro and in vivo uncaging and bioluminescence imaging by using photocaged upconversion nanoparticles," Angewandte Chemie, vol. 51, no. 13, pp. 3125-3129, 2012.

[70] G. Y. Chen, T. Y. Ohulchanskyy, S. Liu et al., "Core/shell $\mathrm{NaGdF}_{4}: \mathrm{Nd}^{3+} / \mathrm{NaGdF}_{4}$ nanocrystals with efficient nearinfrared to near-infrared downconversion photoluminescence for bioimaging applications," ACS Nano, vol. 6, no. 4, pp. 2969-2977, 2012.

[71] J. R. Lindner, "Microbubbles in medical imaging: current applications and future directions," Nature Reviews Drug Discovery, vol. 3, no. 6, pp. 527-532, 2004.

[72] J. Zhong, S. Yang, X. Zheng, T. Zhou, and D. Xing, "In vivo photoacoustic therapy with cancer-targeted indocyanine greencontaining nanoparticles," Nanomedicine, vol. 8, no. 6, pp. 903919, 2013.

[73] K. Wilson, K. Homan, and S. Emelianov, "Biomedical photoacoustics beyond thermal expansion using triggered nanodroplet vaporization for contrast-enhanced imaging," Nature Communications, vol. 3, article 618, 2012.

[74] C. Wu and D. T. Chiu, "Highly fluorescent semiconducting polymer dots for biology and medicine," Angewandte ChemieInternational Edition, vol. 52, no. 11, pp. 3086-3109, 2013.
[75] C. Wu, C. Szymanski, and J. McNeill, "Preparation and encapsulation of highly fluorescent conjugated polymer nanoparticles," Langmuir, vol. 22, no. 7, pp. 2956-2960, 2006.

[76] S. Kim, C. K. Lim, J. Na et al., "Conjugated polymer nanoparticles for biomedical in vivo imaging," Chemical Communications, vol. 46, no. 10, pp. 1617-1619, 2010.

[77] L. Feng, C. Zhu, H. Yuan, L. Liu, F. Lv, and S. Wang, "Conjugated polymer nanoparticles: preparation, properties, functionalization and biological applications," Chemical Society Reviews, vol. 42, no. 16, pp. 6620-6633, 2013.

[78] D. Ding, J. Liu, G. Feng, K. Li, Y. Hu, and B. Liu, "Bright far$\mathrm{red} /$ near-infrared conjugated polymer nanoparticles for in vivo bioimaging," Small, vol. 9, no. 18, pp. 3093-3102, 2013.

[79] E. Ahmed, S. W. Morton, P. T. Hammond, and T. M. Swager, "Fluorescent multiblock $\pi$-conjugated polymer nanoparticles for in vivo tumor targeting," Advanced Materials, vol. 25, no. 32, pp. 4504-4510, 2013.

[80] P. Howes, M. Green, A. Bowers et al., "Magnetic conjugated polymer nanoparticles as bimodal imaging agents," Journal of the American Chemical Society, vol. 132, no. 28, pp. 9833-9842, 2010.

[81] Y.-H. Chan, C. Wu, F. Ye, Y. Jin, P. B. Smith, and D. T. Chiu, "Development of ultrabright semiconducting polymer dots for ratiometric $\mathrm{pH}$ sensing," Analytical Chemistry, vol. 83, no. 4, pp. 1448-1455, 2011.

[82] C. Wu, T. Schneider, M. Zeigler et al., "Bioconjugation of ultrabright semiconducting polymer dots for specific cellular targeting," Journal of the American Chemical Society, vol. 132, no. 43, pp. 15410-15417, 2010.

[83] J. Yu, C. Wu, X. Zhang et al., "Stable functionalization of small semiconducting polymer dots via covalent cross-linking and their application for specific cellular imaging," Advanced Materials, vol. 24, no. 26, pp. 3498-3504, 2012.

[84] Y. Rong, C. Wu, J. Yu et al., "Multicolor fluorescent semiconducting polymer dots with narrow emissions and high brightness," ACS Nano, vol. 7, no. 1, pp. 376-384, 2013.

[85] N. A. A. Rahim, W. McDaniel, K. Bardon et al., "Conjugated polymer nanoparticles for two-photon imaging of endothelial cells in a tissue model," Advanced Materials, vol. 21, no. 34, pp. 3492-3496, 2009.

[86] X. Feng, F. Lv, L. Liu et al., "Conjugated polymer nanoparticles for drug delivery and imaging," ACS Applied Materials and Interfaces, vol. 2, no. 8, pp. 2429-2435, 2010.

[87] B. Sun, M.-J. Sun, Z. Gu et al., "Conjugated polymer fluorescence probe for intracellular imaging of magnetic nanoparticles," Macromolecules, vol. 43, no. 24, pp. 10348-10354, 2010. 


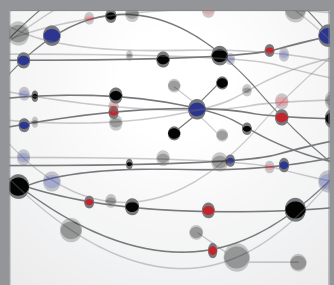

The Scientific World Journal
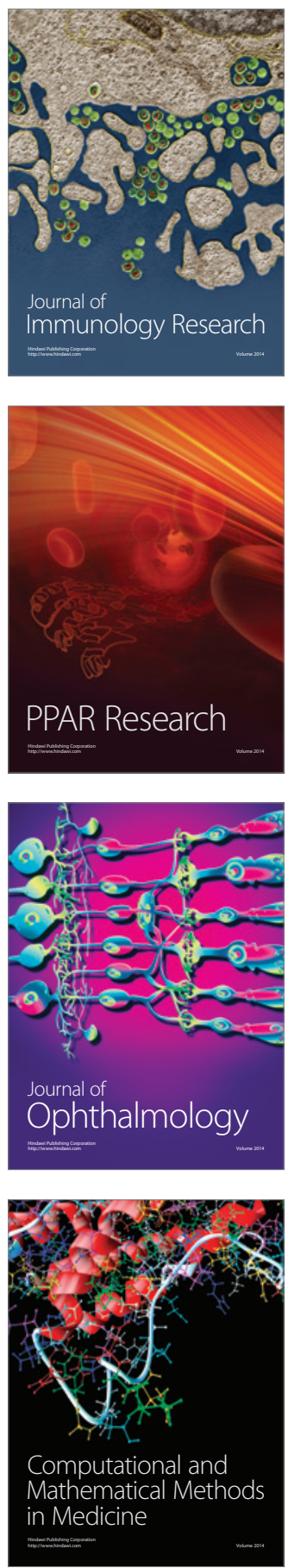

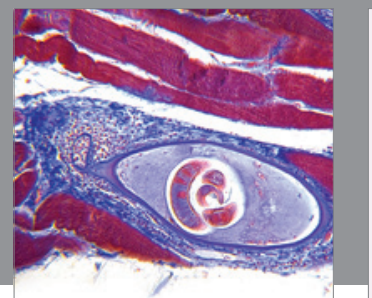

Gastroenterology

Research and Practice
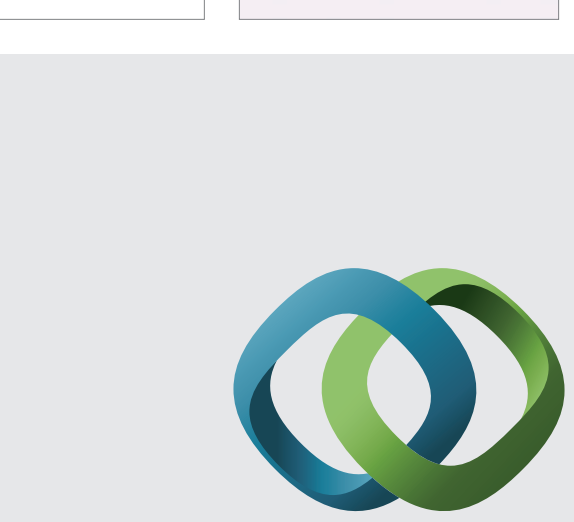

\section{Hindawi}

Submit your manuscripts at

http://www.hindawi.com
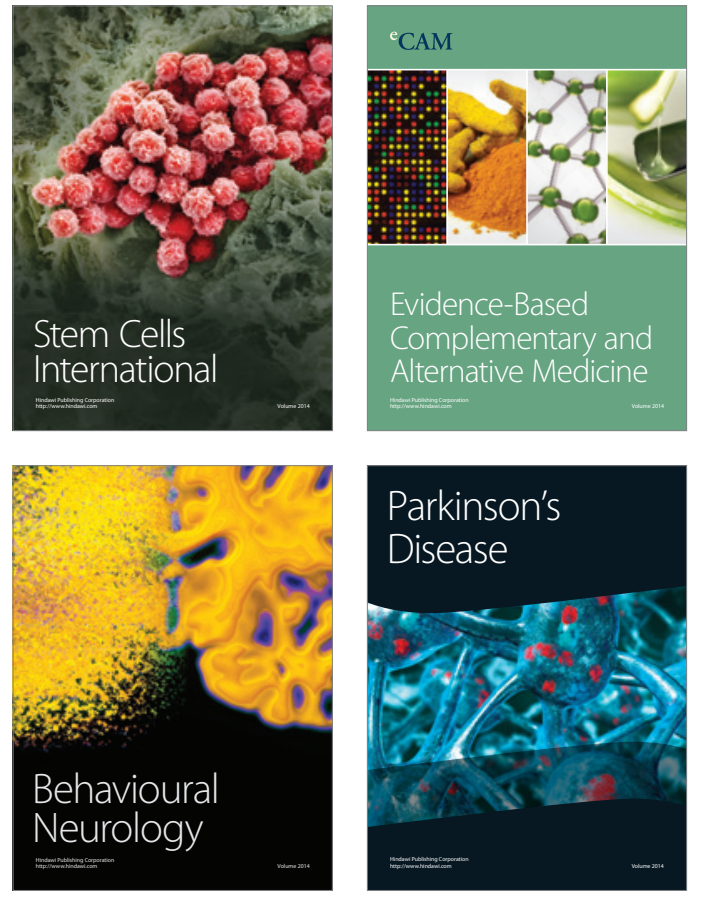
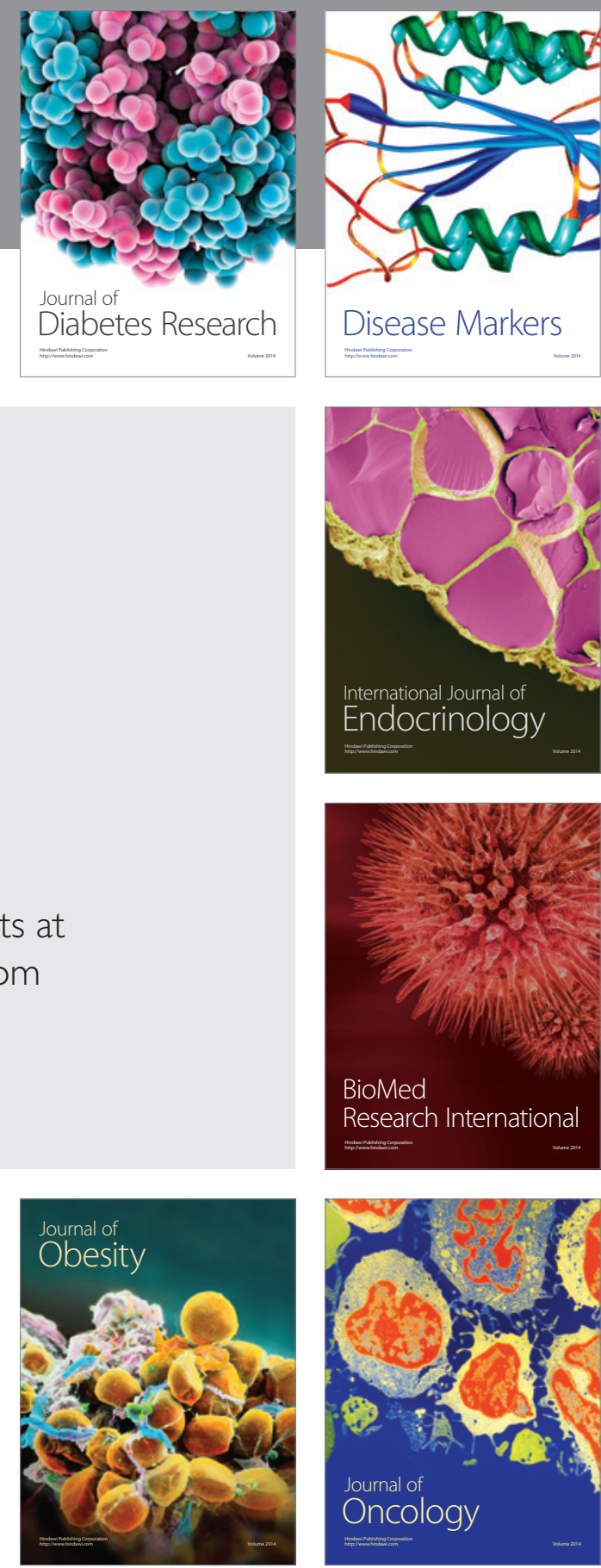

Disease Markers
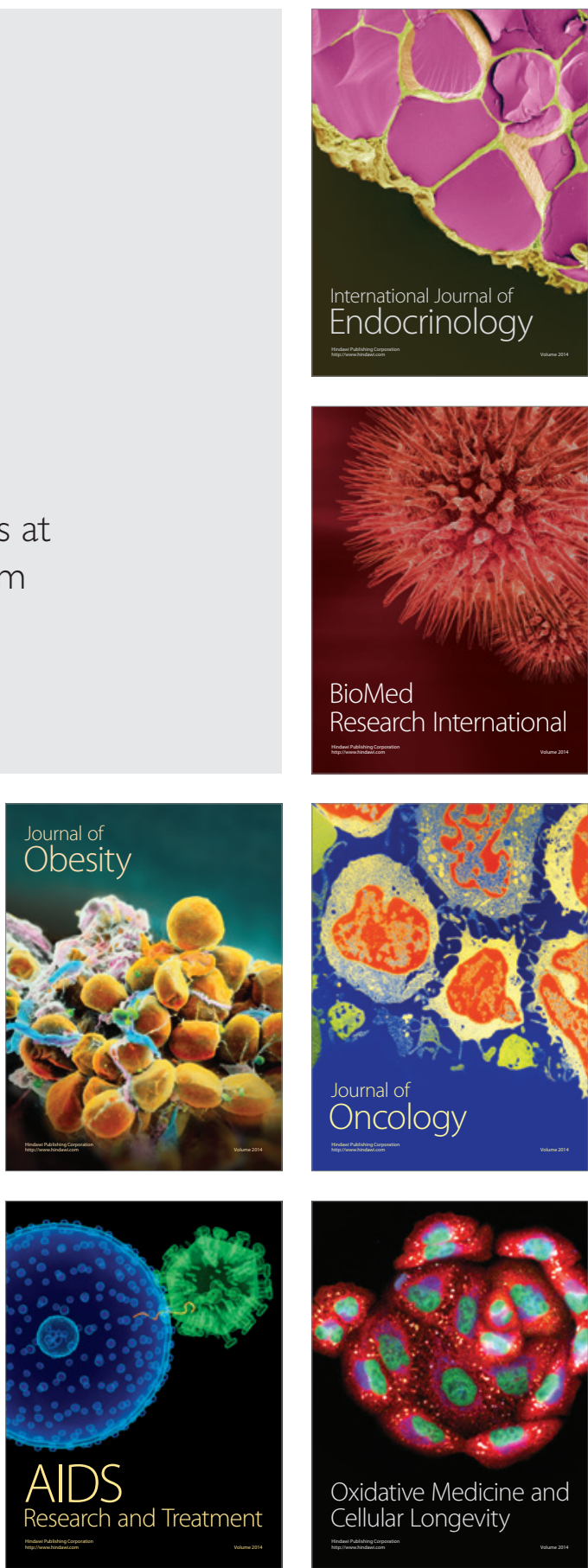